\title{
Raman tensor of graphite: Symmetry of G, D and D' phonons
}

\author{
Mingge Jin $^{\dagger}$, Lu Cheng ${ }^{\dagger}$, Wei Zheng ${ }^{*}$, Ying Ding, Yanming Zhu, Lemin Jia and Feng Huang
}

Graphite is a natural highly-anisotropic layered material formed by stacking in the direction perpendicular to two-dimensional (2D) graphene monolayers. As an anisotropic material, graphite is characterized by its extraordinary optical response [1]. For example, the Raman scattering effect of graphite is both complex and intriguing to be an important window for peeping through its physical properties [1]. Tuinstra et al. [2] reported the Raman spectrum of graphite in 1970 for the first time, and since then a heated discussion has been aroused on the physical origins behind its Raman characteristic peaks [3]. At present, it is generally believed that the Raman active mode $\mathrm{E}_{2 \mathrm{~g} 2}$ at the Brillouin zone $\Gamma$ point corresponding to point group $D_{6 h}$ symmetry results in the so-called first-order Raman $G$ peak with a center at about $1580 \mathrm{~cm}^{-1}$. The Raman features observed at about 1340 and $1620 \mathrm{~cm}^{-1}$ in the defective graphite are defectinduced Raman peaks known as $\mathrm{D}$ and $\mathrm{D}^{\prime}$ peaks, respectively, corresponding to the inter-valley and intra-valley second-order Raman processes, where D peak is ascribed to the in-plane transverse optical (TO) branch near $\mathrm{K}$ point corresponding to $\mathrm{A}_{1}^{\prime}$ mode of point group $D_{3 h}$ symmetry, with $\mathrm{D}^{\prime}$ ascribed to the in-plane longitudinal optical (LO) branch near $\Gamma$ point [3-8]. However, direct experimental evidence to explain the vibration types and phonon symmetry of $\mathrm{D}$ and $\mathrm{D}^{\prime}$ peaks is still in blank, which undoubtedly covers a mysterious veil to these graphite Raman peaks.

Raman spectroscopy is one of the ideal tools to characterize the structure and properties of materials [1,3,4,9-12]. Its angleresolved polarized function can help with a uniform study on the symmetry of materials and Raman tensor. In our previous work, systematic studies have been conducted on the polarized characteristics and phonon symmetry of some typical optically anisotropic materials with aluminum nitride (AlN) [13-16] and transition metal dichalcogenides (TMDs) as the representatives [17-27], from which one method has been proved effective, that is, the information we need such as Raman tensor and differential polarizability can be obtained by fitting the experimental results with theoretical basis suitable for experimental system, so as to explore the vibrational symmetry of material phonon modes.

In this work, experimental results achieved through angle resolved polarized Raman (APR) spectroscopy provide a direct basis for understanding the vibration types and phonon symmetry of graphite Raman peaks. According to our research, we suppose that the $\mathrm{D}^{\prime}$ and $\mathrm{G}$ peaks have the same phonon symmetry, but the $\mathrm{D}$ peak, with a vibration type assigned to $\mathrm{A}_{1}^{\prime}$ mode of the in-plane TO branch, is closely related to the structure of graphite and the symmetry of hexagonal lattice. This research here works as an inspiration to understanding the phonon symmetry of graphite.

A Renishaw micro Raman spectrometer (inVia Reflex) equipped with an $\mathrm{Ar}^{+}$laser which has a power of $50 \mathrm{~mW}$ and a wavelength of $488 \mathrm{~nm}$ and a resolution of $1 \mathrm{~cm}^{-1}$ was used for APR measurement. The emitted light was illuminated on the sample through $50 \times$ Leica micro focusing lens, and then it was collected into the charge-coupled device through the lens, polarimeter, slit and other optical paths for backscattering detection. In order to avoid the sample from being overheating and combustion, test conditions were as follows: exposure time of $1 \mathrm{~s}$, laser power of $5 \%$ and cumulative scanning of 10 times. In order to control the scattering light polarized parallel to the incident light, we inserted a rotatable polarizer into the backscattering light path. The APR spectra were observed using a microscope platform rotating in the direction of incident light. Crystal axis and the experimental system are defined as $a, b, c$ and $x, y, z$, respectively, where the $a(c)$ axis is parallel to the $x(z)$ axis. When the incident light wave vector $\boldsymbol{k}_{i}$ is set along the $c(a)$ axis, it is the $z(x)$ axis that the graphite sample can rotate around with a rotation angle $\omega(\theta)$ between the axis and polarization vector of incident light. By rotating the platform and increasing $\omega(\theta)$, a series of graphite Raman spectra dependent on $\omega(\theta)$ are obtained.

Graphite has a layer structure in which the atoms are arranged in a hexagonal pattern within each layer and the layers are stacked in the $\mathrm{AB}$ sequence. The primitive unit cell of graphite is hexagonal, with dimensions $a=2.47 \AA$ and $c=7.15 \AA$. Graphite has a unit cell consisting of four atoms which bring 12 phonon branches and a space group of $P 6_{3} / m m c$. At $\Gamma$ point, the irreducible expression of the optical mode is [28]:

$\Gamma^{\mathrm{opt}}=\mathrm{A}_{2 \mathrm{u}}+2 \mathrm{~B}_{2 \mathrm{~g}}+\mathrm{E}_{1 \mathrm{u}}+2 \mathrm{E}_{2 \mathrm{~g}}$.

According to the group theory, $\mathrm{A}_{2 \mathrm{u}}$ and $\mathrm{E}_{1 \mathrm{u}}$ modes should be infrared active, $B_{2 g}$ mode should be optically inactive, and only $\mathrm{E}_{2 \mathrm{~g}}$ is Raman active and double degenerate $\left(\mathrm{E}_{2 \mathrm{~g} 1}\right.$ and $\left.\mathrm{E}_{2 \mathrm{~g} 2}\right)$. The Raman tensor of $E_{2 g}$ mode graphite is [29]:

$\boldsymbol{R}\left(\mathrm{E}_{2 \mathrm{~g}}\right):\left(\begin{array}{lll}0 & d & 0 \\ d & 0 & 0 \\ 0 & 0 & 0\end{array}\right),\left(\begin{array}{ccc}-d & 0 & 0 \\ 0 & d & 0 \\ 0 & 0 & 0\end{array}\right)$,

where $d$ is the Raman tensor element. For the hexagonal crystal system, the Raman tensor of $\mathrm{A}^{\prime}{ }_{1}$ mode is expressed as [29]: 
$\boldsymbol{R}\left(\mathrm{A}_{1}^{\prime}\right):\left(\begin{array}{lll}a & 0 & 0 \\ 0 & a & 0 \\ 0 & 0 & b\end{array}\right)$.

In the backscattering APR experiment, two kinds of scattering geometry were used. When measuring the $c$ plane of graphite, the polarized vectors of incident light $\left(\boldsymbol{e}_{\mathrm{i}}\right)$ and scattered light $\left(\boldsymbol{e}_{\mathrm{s}}\right)$ can be expressed as

$\boldsymbol{e}_{\mathrm{i}}=\boldsymbol{e}_{\mathrm{s}}=\left(\begin{array}{c}\cos \omega \\ \sin \omega \\ 0\end{array}\right)$.

Based on classical Placzek approximation [30], the Raman scattering intensity is

$I \propto\left|\boldsymbol{e}_{\mathrm{s}} \cdot \boldsymbol{R} \cdot \boldsymbol{e}_{\mathrm{i}}\right|^{2}$,

and thus, through Equations (2-5), we can obtain:

$I\left(\mathrm{E}_{2 \mathrm{~g}}\right)_{c \text {-plane }}=|d|^{2}$,

$I\left(\mathrm{~A}^{\prime}\right)_{c \text {-plane }}=|a|^{2}$.

When measuring the $m$ plane, there is:

$\boldsymbol{e}_{\mathrm{i}}=\boldsymbol{e}_{\mathrm{s}}=\left(\begin{array}{c}0 \\ \sin \theta \\ \cos \theta\end{array}\right)$,

and thus,

$I\left(\mathrm{E}_{2 \mathrm{~g}}\right)_{m \text {-plane }}=|d|^{2} \sin ^{4} \theta$,

$I\left(\mathrm{~A}^{\prime}\right)_{m \text {-plane }}=|a|^{2} \sin ^{4} \theta+|b|^{2} \cos ^{4} \theta+\frac{1}{2}|a||b| \sin ^{2}(2 \theta)$.

Density-functional theory (DFT) and density-functional perturbation theory (DFPT) were chosen for all the calculations of density functional as used in Vienna $A b$-initio Simulation Package (VASP) code. Besides, ultrasoft pseudopotentials were applied. For the exchange-correlation functional, PerdewBurke-Ernzerhof (PBE) generalized gradient approximation (GGA) [31] was chosen for the analysis. During the whole process of calculation, the plane-wave energy cutoff was set as $450 \mathrm{eV}$, and the force and electronic convergence tolerance were set as $0.001 \mathrm{eV} \AA^{-1}$ and $10^{-7} \mathrm{eV}$, respectively. Monkhorst-Packcentered $k$ grid was set as different sizes for different needs with $16 \times 16 \times 5$ for structure optimization and $20 \times 20 \times 7$ for self-consistent calculation.

The Raman tensor is a symmetric $3 \times 3$ tensor which is relevant to the $j$ th phonon mode and it can be written as $[19,23,30,32]$

$R_{\alpha \beta}(j)=V_{\text {prim }} \sum_{\mu=1}^{N} \sum_{l=1}^{3} \frac{\partial \varepsilon_{\alpha \beta}}{\partial r_{l}(\mu)} \frac{e_{l}^{j}(\mu)}{\sqrt{M_{\mu}}}$.

$V_{\text {prim }}$ stands for the volume of unit cell; $r_{l}(\mu)$ is the $\mu$ th atom's location along direction $l ; M_{\mu}$ represents the $\mu$ th atom's mass.

With the aim to calculate Raman tensor from Equation (11), the first step is to obtain both dynamic matrix $e_{l}^{j}(\mu)$ and derivatives of dielectric tensor $\partial \varepsilon_{\alpha \beta} / \partial r_{l}(\mu)$. The former one can be calculated on the basis of DFPT scheme used in the PHONOPY software [33], which allows an exact (within DFT) computation of phonon frequencies at any Brillouin-zone point. In the DFPT scheme, VASP was used to compute Hellmann-Feynman forces in the $8 \times 8 \times 1$ supercell for both positive and negative atomic displacements, and then the dynamic matrix can be constructed when they were used in PHONOPY software. Through a diagonalization of the matrix, the information like eigenvectors $\mathrm{e}_{l}^{j}(\mu)$ of all $3 N$ normal modes ( $N$ refers to the number of atoms in each unit cell), phonon frequencies and phonon vibrations can be provided. For the latter one, the dielectric tensor derivatives were achieved also by using DFPT scheme in DFT. According to the calculation of this study, the frequency-dependent dielectric tensors should be at $2.54 \mathrm{eV}(488 \mathrm{~nm})$ laser line, a frequency used in the experiment.

Fig. 1a shows the phonon dispersion spectrum of graphite attained through the DFPT diagonalization force constant matrix. The atomic vibrational modes corresponding to G, D and $\mathrm{D}^{\prime}$ peaks are depicted in Fig. $1 \mathrm{~b}$, where carbon atoms are represented by purple balls connected by white bonds, and blue arrows indicate the direction of atomic displacement. All layers are set on the $x y$ plane. For each vibration mode, the phonon symmetry and vibration frequency are also marked in Fig. 1. Graphite owns phonon branches with most of them being degenerate, almost the same as graphene $[3,6,33]$. The DFT phonon calculation above provides some basic information about the physical properties of graphite as a basis for further experimental and theoretical research.

It is generally believed that $\mathrm{D}$ and $\mathrm{D}^{\prime}$ peaks of graphite originate from the double resonance (DR) mechanism induced by defects $[3,4]$, but direct and unequivocal experimental evidence on the vibration types and phonon symmetries corresponding to these Raman peaks is still lacking. It is well known that Raman tensor is useful in showing phonon symmetry $[14,19,23]$.
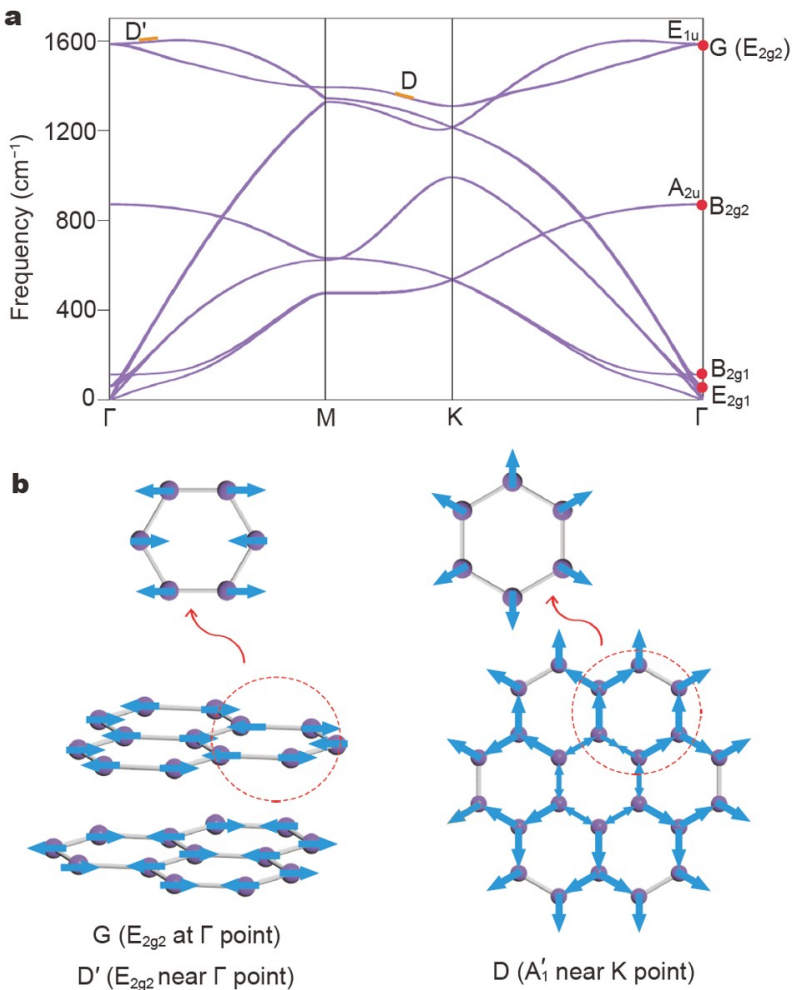

Figure 1 DFT-calculated (a) phonon dispersion relationship and (b) vibration behaviors of graphite [3-5]. The position of each mode is: $\mathrm{E}_{2 \mathrm{~g} 1}$, $60 \mathrm{~cm}^{-1} ; \mathrm{B}_{2 \mathrm{~g} 1}, 113 \mathrm{~cm}^{-1} ; \mathrm{B}_{2 \mathrm{~g} 2}, 870.9 \mathrm{~cm}^{-1} ; \mathrm{A}_{2 \mathrm{u}}, 873 \mathrm{~cm}^{-1} ; \mathrm{E}_{2 \mathrm{~g} 2}, 1581 \mathrm{~cm}^{-1} ; \mathrm{E}_{1 \mathrm{u}}$, $1588 \mathrm{~cm}^{-1}$ 
Therefore, in the following discussion based on the layered structure of graphite, Raman tensor analysis combined with DR mechanism is used to understand the vibration mode and phonon symmetry of the graphite Raman peaks.

Fig. 2b displays the polarized Raman spectra with linearly polarized light incident perpendicular to the $c$ plane of graphite, where the polarized direction of incident light forms a rotation angle $\omega$ varying from $-90^{\circ}$ to $90^{\circ}$ with a step of $10^{\circ}$ to $x$-axis, as shown in Fig. 2a. Raman $G$ peak corresponding to $E_{2 \mathrm{~g} 2}$ mode shows up at about $1579 \mathrm{~cm}^{-1}$, which is close to the DFT calculation result of $1581 \mathrm{~cm}^{-1}$. It can be seen from Fig. $2 \mathrm{~b}, \mathrm{c}$ that the APR intensity of $G$ peak remains as a constant in spite of the change of rotation angle, which conforms to $\mathrm{E}_{2 \mathrm{~g} 2}$ mode in the aspect of Raman selection rule as shown in Equation (6). It is worth noting that since $\mathrm{E}_{2 \mathrm{~g} 2}$ mode belongs to an in-plane vibration mode, its transverse vibration form (TO) now appears under this configuration.

When it comes to $\mathrm{D}$ peak (which refers to $\mathrm{A}^{\prime}{ }_{1}$ mode of $D_{3 h}$ point group), some puzzling situations appear: according to the Raman tensor (Equation (3)) and Raman selection rule of $\mathrm{A}_{1}^{\prime}$ mode (Equation (7)), D peak corresponding to $\mathrm{A}_{1}^{\prime}(\mathrm{LO})$ is expected to turn up under this configuration and then show a constant APR intensity, while now there is only G peak observed in the spectra (Fig. 2b). This situation may be explained as followed: for hexagonal crystal system, $\mathrm{A}_{1}^{\prime}$ mode is polarized along the $c$ axis (corresponding to Raman tensor element $b$ ), but the propagation properties of $\mathrm{A}_{1}^{\prime}$ mode are a bit different for graphite whose structure is formed by stacking discontinuous graphene monolayers through van der Waals force instead of covalent bonds. Therefore, $\mathrm{A}_{1}{ }_{1}$ mode fails to propagate along the $c$ axis (that is, $|b|\left(\mathrm{A}_{1}^{\prime}\right)=0$ for graphite) as that in normal case but can only exist as a transverse vibration form (that is, $\mathrm{A}_{1}{ }_{1}(\mathrm{TO})$ ) in the monolayer. That is to say, even though the in-plane element $a$ in the Raman tensor is not zero, $\mathrm{D}$ peak corresponding to $\mathrm{A}^{\prime}{ }_{1}(\mathrm{LO})$ cannot be detected under this configuration because the structure of graphite is different from that of other typical hexagonal crystals (AlN, $\mathrm{ZnO}$, etc.). Therefore, from the perspective of symmetry and Raman selection rule, the absence of $\mathrm{D}$ peak is directly related to the interlayer discontinuity of graphite material structure during the backscattering measurement of graphite's $c$ plane.

The polarized Raman spectra with linearly polarized light incident perpendicular to the $m$ plane of graphite is shown in Fig. 3b. Under this configuration, the excited G peak now cor- responds to the $\mathrm{LO}$ mode of $\mathrm{E}_{2 \mathrm{~g} 2}$ phonon. The peak position of $\mathrm{G}$ peak in this case is almost the same as that measured perpendicular to the $c$-plane because the Raman measurement can only detect the phonons near $\Gamma$ point, and for graphite, the TO mode and LO mode of $\mathrm{E}_{2 \mathrm{~g} 2}$ phonon are degenerate at that point. At this time, $\mathrm{D}$ peak $\left(\sim 1368 \mathrm{~cm}^{-1}\right)$ and $\mathrm{D}^{\prime}$ peak $\left(\sim 1624 \mathrm{~cm}^{-1}\right)$ are also observed with the latter one shown as a small shoulder near $\mathrm{G}$ peak with a relatively weak intensity compared with that of the former one. The periodic variation of APR intensity for each mode is shown in Fig. 3c. In order to further describe this periodic change, the dependence relationship between the APR intensity of these three distinct Raman modes and the polarization angle is depicted in Fig. $3 \mathrm{~d}-\mathrm{f}$, respectively. It can be noted that when the rotation angle varies from $-90^{\circ}$ to $90^{\circ}$, the strength of these peaks decreases to zero (where $\theta=0^{\circ}$ ) first and then increases with $\theta$ approaching $90^{\circ}$. It is clear that the APR intensity of these three Raman peaks of graphite shows the same polarized angle dependence with all of them being proportional to $\sin ^{4} \theta$. Among these three peaks, the variation of $E_{2 \mathrm{~g} 2}$ phonon is comparatively easier to understand, that is, as shown in Equation (9), it is proportional to $|d|^{2} \sin ^{4} \theta$. However, for $\mathrm{D}^{\prime}$ and $\mathrm{D}$ peaks, the situation is complex with $\mathrm{DR}$ mechanism used for explanation as follows.

According to DR mechanism, D' (D) peak originates from intra-(inter-)valley indirect second-order Raman process with the existence of LO (TO) phonons and defects [4,5]. In order to figure out the polarized angle dependence of these two peaks, phonon symmetry and Raman selective rule were also taken into consideration for a better understanding. Firstly, for $\mathrm{D}^{\prime}$ peak, considering that it appears in the vicinity of $G$ peak, we suppose that it also corresponds to $E_{2 \mathrm{~g} 2}$ phonon like $\mathrm{G}$ peak. The reasons why $\mathrm{D}^{\prime}$ peak cannot be detected during the backscattering measurement of $c$ plane are now clear. On one hand, the detective position is far away from the discontinuity and defect region on the boundary; on the other hand, under this experimental configuration, the TO mode of $\mathrm{E}_{2 \mathrm{~g} 2}$ phonon is excited, which cannot participate in the second-order Raman process of $\mathrm{D}^{\prime}$ peak. On the contrary, during the backscattering measurement of $m$ plane, the boundary region is directly detected with $\mathrm{LO}$ mode of $\mathrm{E}_{2 \mathrm{~g} 2}$ phonon excited, and thus $\mathrm{D}^{\prime}$ peak can appear. For a further step, according to the Raman tensor of $E_{2 \mathrm{~g} 2}$ mode, it can be found that the Raman intensity of $\mathrm{D}^{\prime}$ peak is proportional to $|d|^{2} \sin ^{4} \theta$. Similarly, for $\mathrm{D}$ peak corresponding to $\mathrm{A}^{\prime}{ }_{1}$ phonon, $\mathrm{A}_{1}{ }_{1}(\mathrm{TO})$ is excited during the backscattering measurement of $m$ plane. Accordingly, if the Raman tensor element $|b|$ of
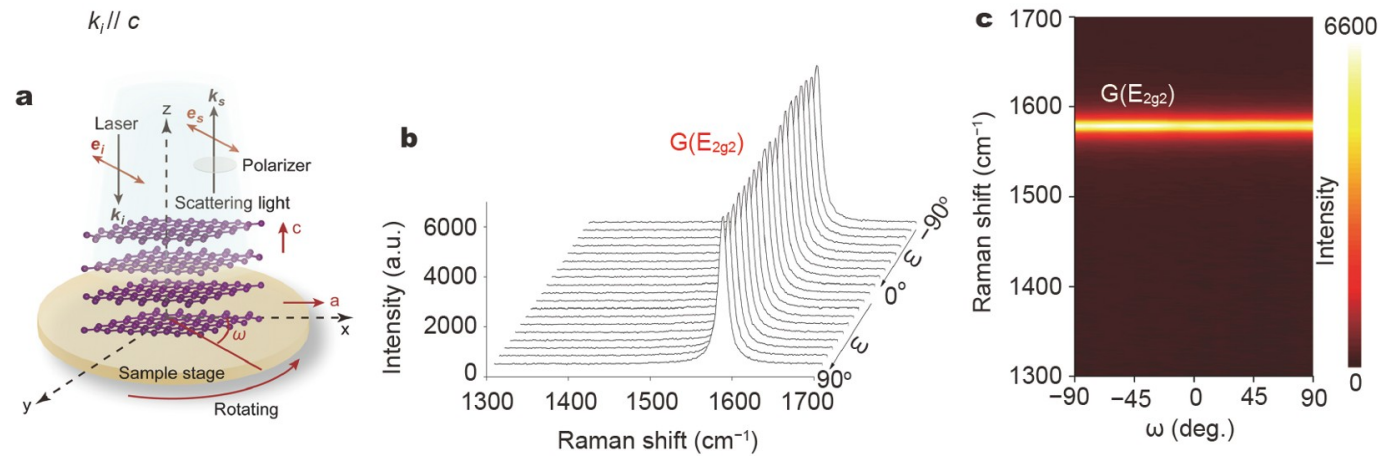

Figure 2 APR spectroscopy of the $c$ plane of graphite. (a) Diagram of the measurement configuration. (b) Polarized Raman spectra of graphite for the $c$ plane surface. (c) False-color plot that corresponds to the polarized Raman spectra. The color scale in the plot represents the intensity of Raman vibration. 

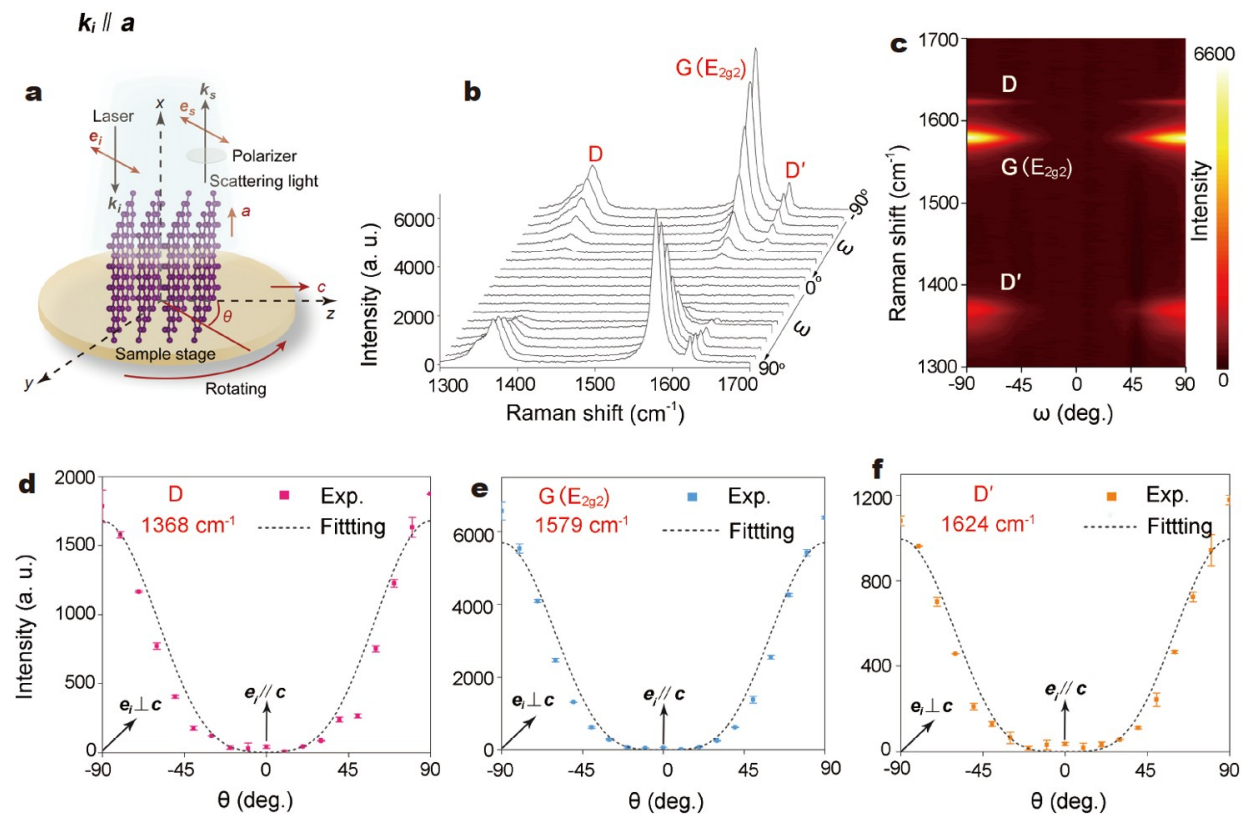

Figure 3 APR spectroscopy of $m$ plane of graphite. (a) Diagram of the measurement configuration. (b) Polarized Raman spectra of graphite for $m$-plane surface. (c) False-color plot that corresponds to the polarized Raman spectra. The color scale in the plot represents the intensity of Raman vibration. (d-f) Angle dependence of Raman intensity of D, G and D' peaks. Colored triangles represent the experimental data, and black dotted lines are the fitting lines.

Table 1 Raman tensors of graphite vibration modes achieved through APR scattering spectrum and the first-principles calculation

\begin{tabular}{|c|c|c|}
\hline Mode & Theoretical Raman tensors & Experimental Raman tensors \\
\hline$E_{2 g 1}$ & $0.01\left(\begin{array}{ccc}-1 & 1 & 0 \\
1 & 1 & 0 \\
0 & 0 & 0\end{array}\right)$ & l \\
\hline $\mathrm{D}\left(\mathrm{A}_{1}^{\prime}\right.$ near $\mathrm{K}$ point $)$ & l & $0.8\left(\begin{array}{lll}1 & 0 & 0 \\
0 & 1 & 0 \\
0 & 0 & 0\end{array}\right)$ \\
\hline $\mathrm{G}\left(\mathrm{E}_{2 \mathrm{~g} 2}\right.$ at $\Gamma$ point $)$ & $1.5 e^{(-0.25 \pi i)}\left(\begin{array}{ccc}-1 & 1 & 0 \\
1 & 1 & 0 \\
0 & 0 & 0\end{array}\right)$ & $1.5\left(\begin{array}{ccc}-1 & 1 & 0 \\
1 & 1 & 0 \\
0 & 0 & 0\end{array}\right)$ \\
\hline $\mathrm{D}^{\prime}\left(\mathrm{E}_{2 \mathrm{~g} 2}\right.$ near $\Gamma$ point $)$ & l & $0.6\left(\begin{array}{ccc}-1 & 1 & 0 \\
1 & 1 & 0 \\
0 & 0 & 0\end{array}\right)$ \\
\hline
\end{tabular}

Note: Considering that the relative values of Raman intensity have the significance of comparison, and in order to facilitate the comparison between theoretical and experimental results, we take the Raman tensor element $|d|\left(\mathrm{D}^{\prime}\right)$ of the experimentally measured $\mathrm{G}$ peak as 1.5 , and convert the tensor elements of $\mathrm{D}$ and $\mathrm{D}^{\prime}$ peaks by equal proportion, that is, $|a|(\mathrm{D})=0.8$ and $|d|\left(\mathrm{D}^{\prime}\right)=0.6$, respectively.

$\mathrm{A}^{\prime}{ }_{1}$ mode is takenas zero, the Raman intensity of $\mathrm{D}$ peak will be proportional to $|a|^{2} \sin ^{4} \theta$ [34]. The meaning of $|b|$ being taken as zero corresponds to $\mathrm{A}_{1}^{\prime}$ mode which cannot propagate along the $c$ axis of graphite. The conclusion obtained directly from the APR experiment here is consistent with the theoretical calculations by Piscanec et al. [6], that is, the D peak comes from $\mathrm{A}_{1}^{\prime}(\mathrm{TO})$.

According to the idea above, Equations $(9,10)$ were taken to fit the Raman intensities of these modes (see black dotted line in Fig. 3d-f), and thus the Raman tensor elements were obtained as follows: $\quad|d|\left(\mathrm{D}^{\prime}\right)=31.5, \quad|d|(\mathrm{G})=75.5, \quad|a|(\mathrm{D})=40.7, \quad|b|(\mathrm{D})=0$, respectively. In addition, the Raman tensors of Raman-active mode were also calculated under $488 \mathrm{~nm}$ laser line excitation, according to which the results including ab-initio Raman tensor form of Raman-active $E_{2 g 1}$ and $E_{2 g 2}$ modes were obtained with their calculated Raman tensor element $d$ listed in Table 1 . The calculated Raman tensor form of $\mathrm{E}_{2 \mathrm{~g} 2}$ mode corresponding to the $\mathrm{G}$ peak of graphite is consistent with the experimental one, which proves the correctness of the experimental results.

In summary, this work studies the phonon symmetry and Raman tensor of graphite Raman peaks through experimental APR spectroscopy directly and systematically. According to this research, $D^{\prime}$ and $G$ peaks are the same in the aspect of phonon 
symmetry, that is, $\mathrm{E}_{2 \mathrm{~g} 2}(\mathrm{LO})$, while $\mathrm{D}$ peak corresponding to $\mathrm{A}_{1}^{\prime}(\mathrm{TO})$ symmetry is closely related to the specific crystal structure of graphite. The findings work as a direct and powerful experimental basis for better understanding on the phonon symmetry graphite Raman peaks.

Received 5 April 2021; accepted 17 June 2021; published online 6 August 2021

1 Ferrari AC. Raman spectroscopy of graphene and graphite: Disorder, electron-phonon coupling, doping and nonadiabatic effects. Solid State Commun, 2007, 143: 47-57

2 Tuinstra F, Koenig JL. Raman spectrum of graphite. J Chem Phys, 1970, 53: $1126-1130$

3 Ferrari AC, Basko DM. Raman spectroscopy as a versatile tool for studying the properties of graphene. Nat Nanotech, 2013, 8: 235-246

4 Malard LM, Pimenta MA, Dresselhaus G, et al. Raman spectroscopy in graphene. Phys Rep, 2009, 473: 51-87

5 Beams R, Gustavo Cançado L, Novotny L. Raman characterization of defects and dopants in graphene. J Phys-Condens Matter, 2015, 27: 083002

6 Piscanec S, Lazzeri M, Mauri F, et al. Kohn anomalies and electronphonon interactions in graphite. Phys Rev Lett, 2004, 93: 185503

7 Maultzsch J, Reich S, Thomsen C, et al. Phonon dispersion in graphite. Phys Rev Lett, 2004, 92: 075501

8 Jiang J, Saito R, Samsonidze GG, et al. Electron-phonon matrix elements in single-wall carbon nanotubes. Phys Rev B, 2005, 72: 235408

9 Strach T, Brunen J, Lederle B, et al. Determination of the phase difference between the Raman tensor elements of the $\mathrm{A}_{1 \mathrm{~g}}$-like phonons in $\mathrm{SmBa}_{2} \mathrm{Cu}_{3} \mathrm{O}_{7-\delta}$. Phys Rev B, 1998, 57: 1292-1297

10 Tsukada S, Fujii Y, Yoneda Y, et al. Raman scattering study of the ferroelectric phase transition in $\mathrm{BaTi}_{2} \mathrm{O}_{5}$. Phys Rev B, 2018, 97: 024116

11 Livneh T, Zhang J, Cheng G, et al. Polarized Raman scattering from single GaN nanowires. Phys Rev B, 2006, 74: 035320

12 Ribeiro HB, Pimenta MA, de Matos CJS, et al. Unusual angular dependence of the Raman response in black phosphorus. ACS Nano, 2015, 9: 4270-4276

13 Cheng L, Zheng W, Jia L, et al. Quasiphonon polaritons. Heliyon, 2020, 6: e05277

14 Zheng W, Zheng R, Huang F, et al. Raman tensor of AlN bulk single crystal. Photon Res, 2015, 3: 38-43

15 Zheng W, Lin R, Ran J, et al. Vacuum-ultraviolet photovoltaic detector. ACS Nano, 2018, 12: 425-431

16 Jia L, Zheng W, Huang F. Vacuum-ultraviolet photodetectors. PhotoniX, 2020, 1: 22

17 Zheng W, Li F, Li G, et al. Laser tuning in van der Waals crystals. ACS Nano, 2018, 12: 2001-2007

18 Zheng W, Zhu Y, Li F, et al. Raman spectroscopy regulation in van der Waals crystals. Photon Res, 2018, 6: 991-995

19 Jin M, Zheng W, Ding Y, et al. Raman tensor of $\mathrm{WSe}_{2}$ via angleresolved polarized Raman spectroscopy. J Phys Chem C, 2019, 123: 29337-29342

20 Ding $\mathrm{Y}$, Zheng W, Jin M, et al. Raman tensor of layered $\mathrm{MoS}_{2}$. Opt Lett, 2020, 45: 1313-1316

21 Ding Y, Zheng W, Lin Z, et al. Raman tensor of layered $\mathrm{WS}_{2}$. Sci China Mater, 2020, 63: 1848-1854

22 Ding Y, Zheng W, Zhu Y, et al. Raman tensor of layered Td-WTe 2 . J Phys Chem C, 2020, 124: 16596-16603

23 Jin M, Zheng W, Ding Y, et al. Raman tensor of van der Waals MoSe $e_{2}$. J Phys Chem Lett, 2020, 11: 4311-4316

24 Zhu Y, Zheng W, Wang W, et al. Raman tensor of layered black arsenic. J Raman Spectrosc, 2020, 51: 1324-1330

25 Ding Y, Zheng W, Lu X, et al. Raman tensor of layered $\mathrm{SnS}_{2}$. J Phys Chem Lett, 2020, 11: 10094-10099

26 Zhu Y, Zheng W, Wang W, et al. Raman tensor of layered black phosphorus. PhotoniX, 2020, 1: 17

27 Ding Y, Zheng W, Zhu Y, et al. Laser tuning in layered h-BN crystals. J
Phys Chem Lett, 2021, 12: 3795-3801

28 Nemanich RJ, Lucovsky G, Solin SA. Infrared active optical vibrations of graphite. Solid State Commun, 1977, 23: 117-120

29 Loudon R. The Raman effect in crystals. Adv Phys, 1964, 13: 423-482

30 Cardona M, Güntherodt G. Light Scattering in Solids II. Optica Acta. Berlin: Springer, 1982, 1-14

31 Perdew JP, Burke K, Ernzerhof M. Generalized gradient approximation made simple. Phys Rev Lett, 1996, 77: 3865-3868

32 Massote DVP, Liang L, Kharche N, et al. Electronic, vibrational, Raman, and scanning tunneling microscopy signatures of two-dimensional boron nanomaterials. Phys Rev B, 2016, 94: 195416

33 Baroni S, de Gironcoli S, dal Corso A, et al. Phonons and related crystal properties from density-functional perturbation theory. Rev Mod Phys, 2001, 73: 515-562

34 Li Z, Young RJ, Kinloch IA, et al. Quantitative determination of the spatial orientation of graphene by polarized Raman spectroscopy. Carbon, 2015, 88: 215-224

Acknowledgements This work was financially supported by the National Natural Science Foundation of China (91833301 and 61427901).

Author contributions Zheng $\mathrm{W}$ and Huang $\mathrm{F}$ designed and directed this study. Jin $\mathrm{M}$ and Cheng L led the experimental work, DFT simulations and paper preparation. Ding Y, Zhu Y, Jia L contributed to experimental analysis. The authors read and approved the final manuscript.

Conflict of interest The authors declare no conflict of interest.

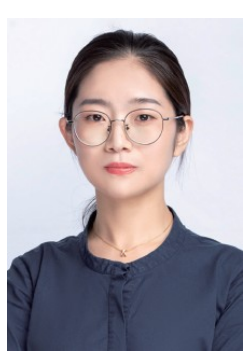

Mingge Jin received her PhD degree from Sun Yatsen University in 2018. Now she is an assistant researcher at the School of Materials, Sun Yat-sen University. Her current scientific research direction is mainly on the Raman tensor of van der Waals layered materials.

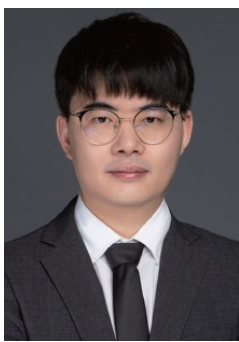

Wei Zheng received his $\mathrm{PhD}$ degree from Shenzhen University in 2014. Now he is a professor at the School of Materials, Sun Yat-sen University. His research interest focuses on semiconductor-based vacuum-ultraviolet $(10-200 \mathrm{~nm})$ photodetectors and condensed matter physics in ultra-wide bandgap semiconductors.

\section{石墨的拉曼张量: G、D和 $\mathrm{D}^{\prime}$ 声子的对称性}

金明革 ${ }^{\dagger}$, 程璐 ${ }^{\dagger}$, 郑伟 ${ }^{*}, 丁$ 莹, 朱燕明, 贾乐敏, 黄丰

摘要 自石墨的拉曼光谱在 1970 年被首次记录以来, 其拉曼特征峰 (G、 $\mathrm{D}$ 和 $\mathrm{D}^{\prime}$ 峰) 背后的物理起源一直是人们争论的焦点. 目前, 人们普 遍认为 $G$ 峰对应于拉曼活性 $E_{2 \mathrm{~g} 2}$ 模式, $D$ 和 $\mathrm{D}^{\prime}$ 峰则是与缺陷有关的无序 诱发峰. 然而, 到目前为止, 这些石墨拉曼峰所对应的声子对称性, 还 缺乏细致的研究与直接的实验证据. 在这里, 我们利用角分辨偏振拉曼 光谱对这些重要方面进行了阐述. 结果表明, $\mathrm{D} 、 \mathrm{D}^{\prime}$ 峰的实验拉曼强度 与 $\mathrm{G}$ 峰的偏振角关系相似. 结合拉曼张量分析和双共振机制, 我们进一 步了解了 $\mathrm{D}^{\prime}$ 和 $\mathrm{D}$ 峰的声子对称性. 我们的工作为石墨声子对称性提供了 可靠的实验证据和合理的解释. 